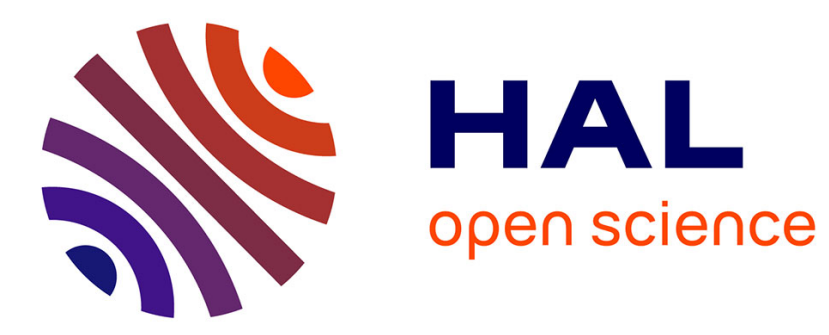

\title{
Filtering Voluntary Motion for Pathological Tremor Compensation
}

\author{
Antonio Bo, Philippe Poignet, Christian Geny
}

\section{To cite this version:}

Antonio Bo, Philippe Poignet, Christian Geny. Filtering Voluntary Motion for Pathological Tremor Compensation. IROS: Intelligent RObots and Systems, Oct 2009, St. Louis, MO, United States. pp.55-60, 10.1109/IROS.2009.5353972 . lirmm-00412950

\section{HAL Id: lirmm-00412950 https://hal-lirmm.ccsd.cnrs.fr/lirmm-00412950}

Submitted on 2 Sep 2009

HAL is a multi-disciplinary open access archive for the deposit and dissemination of scientific research documents, whether they are published or not. The documents may come from teaching and research institutions in France or abroad, or from public or private research centers.
L'archive ouverte pluridisciplinaire HAL, est destinée au dépôt et à la diffusion de documents scientifiques de niveau recherche, publiés ou non, émanant des établissements d'enseignement et de recherche français ou étrangers, des laboratoires publics ou privés. 


\title{
Filtering Voluntary Motion for Pathological Tremor Compensation
}

\author{
Antônio Padilha Lanari Bó, Philippe Poignet, and Christian Geny
}

\begin{abstract}
This paper describes an algorithm to estimate tremor and voluntary motion from measured motion data. Estimation is performed by means of an extended Kalman filter (EKF), which also estimates tremor parameters. Comparison of the proposed method with techniques described in the literature are conducted with two experimental data sets from tremor patients performing the same task, drawing a spiral. The presented algorithm may be directly applied in real-time pathological tremor compensation systems.
\end{abstract}

\section{INTRODUCTION}

Tremor may be defined as an involuntary, approximately rhythmic and roughly sinusoidal movement [1]. Although pathological tremor itself is not lifethreatening, it may decrease considerably the person's quality of life, since the ability to perform simple daily tasks is reduced. It is the most common movement disorder found in human pathology.

An absolutely effective treatment for pathological tremor is not yet available. Although pharmacological and surgical therapies are available, they still present limitations with reference to cost, risks, and effectiveness. In this scenario, one alternative is the use of assistive devices to reduce the effects of the abnormal motion. Among the new technologies, upper limb robotic exoskeletons [2] and the use of FES [3] have been proposed.

Within the context of assistive devices to attenuate tremor, every candidate technology must continuously estimate tremor during the device operation. This is due to the fact that pathological tremor presents high variability throughout time, particularly in terms of tremor amplitude. Our effort to address this problem has been described in [4], where an algorithm to perform online tremor characterization based on two distinct parametric models was presented.

However, the sensors used to measure the current tremor state are also sensitive to the voluntary motion performed by the patient, which is normally executed in lower frequencies, when compared to pathological tremor frequencies. Hence, in order to correctly estimate the tremor motion, the voluntary component must be extracted from the total movement.

Some methods have already been proposed to address this problem. [5] presented the application of digital

Antônio P. L. Bó (e-mail: bo@lirmm.fr) and Philippe Poignet are with the LIRMM UMR 5506 CNRS UM2, France. Antônio P. L. Bó is also with Neuromedics, France. Christian Geny is with the CHU Montpellier, France. filters to suppress tremor from computer input, but the method is not feasible for a real-time compensation system. Regarding methods applied to real-time compensation problems, in [6] an adaptive filter was used to estimate tremor modeled as an harmonic model. The same algorithm was also applied in [2]. In that paper, however, explicit estimation of voluntary motion was added.

In the current paper, a new technique to perform tremor and voluntary motion estimation is presented. The algorithm was designed to improve the filter robustness to multiple motions and different sensors. To accomplish that, models are assumed for both tremor and intentional motion and an extended Kalman filter (EKF) is used as the recursive estimation algorithm. The EKF presents the advantage of explicitly considering the measurement noise and concurrently performing estimation of the tremor and voluntary motion.

The assumption of an explicit voluntary motion model may also be important in the estimation of a particular motion or task in order to trigger a compensation pattern. It may also be an useful feature for other applications. For instance, a good estimation of intentional motion may be important in gesture recognition or different rehabilitation therapies involving tremor patients.

The paper is organized as follows. In the following section our EKF-based solution to the problem is described, including description of tremor and voluntary motion models, the estimation algorithm and parameters selection. Section III presents the experimental evaluation of the proposed method with two different data sets. The experimental data was obtained from tremor patients performing the same task, but measured with different sensors. This section also presents a comparison with the results provided by the techniques proposed in [6], [2]. The last section presents the final remarks.

\section{Estimating TREMOR AND VOLUNTARY MOTION}

It is considered in this paper that the motion performed by the patient is composed by pathological tremor and voluntary motion. Both signals are nonstationary. For the scalar case, the total motion is given by

$$
s(k)=s_{t}(k)+s_{i}(k),
$$

where $k$ is a multiple of $T$, the sampling period, $s_{t}$ is the tremor component, and $s_{i}$ is the intentional motion. $s$ is measured by a motion sensor, which may be an inertial 
sensor, an optical tracking system, digitizing tablets or similar devices. The sensor provides the measurement

$$
\tilde{s}(k)=s(k)+\nu_{s}(k),
$$

where $\nu_{s}$ is an additive white Gaussian noise, $\nu_{s} \sim$ $N\left(0, \sigma_{s}^{2}\right)$, that represents sensor error.

Our goal in this work is to estimate online both tremor and voluntary motion components from the noisy measurements of a motion sensor. In the following subsection we describe the models adopted for each separate motion. Following, the recursive algorithm used to estimate the referred signal and the tremor parameters is presented.

\section{A. Models}

Since we are estimating both voluntary and tremor motions from the measured resultant motion, our knowledge from these different motions must be employed to separate them. We assume that voluntary motion lies mainly within low frequencies $(<2 \mathrm{~Hz})$, while tremor frequencies are higher (roughly between 3 to $15 \mathrm{~Hz}$ ).

In this scenario, one may simply choose to apply classic linear filters, but this option was not considered due to two main reasons. Firstly, the intrinsic delays added are not desirable, since the estimated tremor motion will be used by a closed-loop tremor compensation system. Furthermore, since we are interested in estimating in real-time the tremor model parameters, a model-based estimation procedure must be applied in any case.

The other alternative is to describe the motions of interest by general mathematical models, which then are used in the estimation procedure. In [6], for instance, an harmonic model is assumed for tremor, while no explicit model for intentional motion is assumed. In [2], models for both motions are assumed, which is also the chosen approach in this work.

Regarding the tremor model, in [4], we have compared the use of Auto-Regressive (AR) and harmonic models to the problem of tremor online estimation only. The cited paper did not discard the use of a particular model, since both models presented good performance for tremor estimation, including the estimation of power spectrum and $k$-step ahead prediction. For the present case, however, since two motions are estimated simultaneously, harmonic models were chosen as the tremor model. This was mainly due to the fact that the parameters that describe the frequency in harmonic models may be explicitly bounded, while in AR models the frequency behavior is described by the combination of the different parameters. Hence, filter convergence to consistent parameters become less robust.

An harmonic model may represent any periodic signal. In the case of quasi-periodic signals, an approximation may be achieved with harmonic models. Since a nonstationary signal is considered, the following rectangular model is adopted for unidimensional motion:

$$
\begin{array}{r}
s_{t}(k)=\sum_{h=1}^{H}\left[a_{h}(k) \sin \left(h \sum_{t=1}^{k} \omega(t)\right)+\right. \\
\left.b_{h}(k) \cos \left(h \sum_{t=1}^{k} \omega(t)\right)\right]+\nu_{s_{t}}(k),
\end{array}
$$

where $\omega$ is the fundamental frequency, $a_{h}$ and $b_{h}$ are the coefficients and $H$ is the number of harmonics, the model order. $\nu_{s t}$, an additive white Gaussian noise, $\nu_{s_{t}} \sim$ $N\left(0, \sigma_{s_{t}}^{2}\right)$, represents modeling errors. For motions in $\Re^{2}$ or $\Re^{3}$, another advantage of harmonic models is that coupling between each coordinate may be represented by assuming that every tremor motion, modeled by (3), has the same fundamental frequency $\omega$.

In this model, it is assumed that tremor may be described by harmonic frequencies, which is often the case. For motions that do not present this feature, one option is to adopt nonharmonic relations between the frequencies, as in [7]. The algorithm presented in this paper may be easily adapted to this nonharmonic model.

Concerning the voluntary motion, although it is a slower movement, it does not present the regular features of the tremor motion. Hence, in this work it was modeled as low-pass filtered white noise:

$$
s_{i}(k)=\sum_{f=1}^{F} c_{f} \nu_{s_{i}}(k-f),
$$

where $\nu_{s t}$ is a white Gaussian noise, $\nu_{s_{i}} \sim N\left(0, \sigma_{s_{i}}^{2}\right)$, and $F$ is the model order. $c_{f}$, the filter parameters, are fixed parameters tuned to represent the frequency behavior assumed for voluntary motion, i.e., a low-pass filter with cutoff frequency around $2 \mathrm{~Hz}$.

\section{B. $E K F$}

Some algorithms were already proposed to perform the separation of tremor and voluntary motion. The Weighted Fourier Linear Combined (WFLC), described in [6], is an algorithm designed to estimate an harmonic tremor model, but may be also used to separate tremor and voluntary motion. The estimation problem is solved in two steps: the tremor frequency is estimated from the bandwidth filtered signal and the other parameters are estimated from the raw signal, $\tilde{s}$. A constant term is added in the harmonic model in order to represent voluntary motion. This solution is highly dependent on the chosen gains of the recursive estimation algorithm for different voluntary motions and sensor noises.

In order to address these problems, in [2] another solution was proposed. Voluntary motion is firstly estimated from the original signal by a Benedict-Bordner filter $(\mathrm{BBF})$, which provides $\hat{s}_{i}$. After, the WFLC algorithm is applied to the signal $\tilde{s}-\hat{s}_{i}$. The BBF is equivalent to the Kalman filter steady-state solution to a linear first-order system, but with fixed restrictions between the filter parameters. In this case, considering that voluntary 
motion generally does not behave as the assumed model and that the correction is given by the total measured motion, the result is similar to apply a low-pass filter to estimate $\hat{s}_{i}$, and then apply the WFLC algorithm to estimate $\hat{s}_{t}$.

In our approach, we tried to address the disadvantages of previous solutions. Hence, not only models for both motions are assumed, but also the models uncertainties are explicitly considered. Furthermore, measurement error is represented, resulting in a more robust solution. The optimal estimator for such problems is the Kalman filter (KF) for the linear case. Since the present problem is nonlinear, one alternative is to use a modification of the KF for nonlinear systems, the EKF, where Kalman equations are applied to the first-order linearization of the nonlinear system [8].

The applied filter simultaneously estimates both referred motions. The filter states are composed by those states related to tremor, $\mathbf{x}_{t}$, which are composed by the estimated motion and the tremor model recursively identified parameters,

$$
\left[\begin{array}{llllllll}
s_{t}(k) & a_{1}(k) & \cdots & a_{H}(k) & b_{1}(k) & \cdots & b_{H}(k) & \omega(k)
\end{array}\right]^{T}
$$

and the states related to voluntary motion, $\mathbf{x}_{i}$, which are organized accordingly,

$$
\left[\begin{array}{llllll}
s_{i}(k) & \cdots & s_{i}(k-f) & \nu_{s_{i}}(k-1) & \cdots & \nu_{s_{i}}(k-f)
\end{array}\right]^{T} .
$$

To obtain the full state vector, we combine the states regarding tremor and voluntary motion:

$$
\mathbf{x}(k)=\left[\begin{array}{l}
\mathbf{x}_{t}(k) \\
\mathbf{x}_{i}(k)
\end{array}\right] .
$$

The total number of states will be defined by the orders chosen for both motion models:

$$
n=1+(2 H+1)+2 F .
$$

In the KF framework, estimation is organized in prediction and correction phases. In the prediction phase, $\hat{\mathbf{x}}^{-}(k)$, the initial a priori estimate is computed according to

$$
\hat{\mathbf{x}}^{-}(k)=f(\hat{\mathbf{x}}(k-1)),
$$

where $f(\mathbf{x})$ is composed by Eqs. (3) and (4) and the parameters models. All parameters are modeled as random walk processes, particularly since they do present fast dynamics. For instance, the evolution of parameter $\omega$ is given by

$$
\omega(k)=\omega(k-1)+\nu_{\omega}(k),
$$

where $\nu_{\omega}$ is a white Gaussian noise, $\nu_{\omega} \sim N\left(0, \sigma_{\omega}^{2}\right)$.

The error covariance matrix, $\mathbf{P}$, is propagated according to

$$
\mathbf{P}^{-}(k)=\mathbf{A} \mathbf{P}(k-1) \mathbf{A}^{T}+\mathbf{Q},
$$

where $\mathbf{A}$ is the jacobian matrix of $\mathbf{f}(\hat{\mathbf{x}}(k-1))$ with respect to $\hat{\mathbf{x}}(k-1)$ and $\mathbf{Q}$, the process covariance matrix, is a diagonal matrix whose components are the variances of each filter state.

In the correction phase, the available measurement,

$$
\mathbf{y}(k)=g(\mathbf{x}(k)),
$$

where $g(\mathbf{x}(k))$ is given by Eqs. (1) and (2), is used to correct the initial estimate, providing $\hat{\mathbf{x}}^{+}(k)$, the final a posteriori estimate. The Kalman gain, $K$, scales the correction applied in the correction phase:

$$
K(k)=\mathbf{P}^{-}(k) \mathbf{C}^{T}\left(\mathbf{C P}^{-}(k) \mathbf{C}^{T}+r\right)^{-1},
$$

where $\mathbf{C}$ is the jacobian matrix of $\mathbf{g}\left(\hat{\mathbf{x}}^{-}(k)\right)$ with respect to $\hat{\mathbf{x}}^{-}(k)$. Once it is computed, the final estimate is computed according to

$$
\hat{\mathbf{x}}^{+}(k)=\hat{\mathbf{x}}^{-}(k)+K(k)\left(\mathbf{y}(k)-\mathbf{g}\left(\hat{\mathbf{x}}^{-}(k)\right)\right)
$$

and the corresponding error covariance matrix is given by

$$
\mathbf{P}(k)=(\mathbf{I}-K(k) \mathbf{C}) \mathbf{P}^{-}(k),
$$

where $\mathbf{I}$ is the identity matrix.

Regarding the parameters that may be tuned in the algorithm, another advantage of the EKF is that the filter parameters have a direct physical meaning. They are related to the associated uncertainties and to the initial conditions. The process covariance matrix, $\mathbf{Q}$, represents the uncertainties related to the motion models and the models parameters. Variances related to the models are lower for imprecise models, while variances related to parameters are proportional to the time-varying nature of those parameters. As to the measurement variance $r$, it is considered equal to the variance $\sigma_{s}^{2}$.

Concerning the initial conditions, the initial frequency estimate is critical. Fortunately, to obtain a good estimate is not an unusual procedure, being normally conducted in clinical settings. The other parameters are initialized as zero. The initial error covariance matrix, $\mathbf{P}$, have similar values of corresponding states variances.

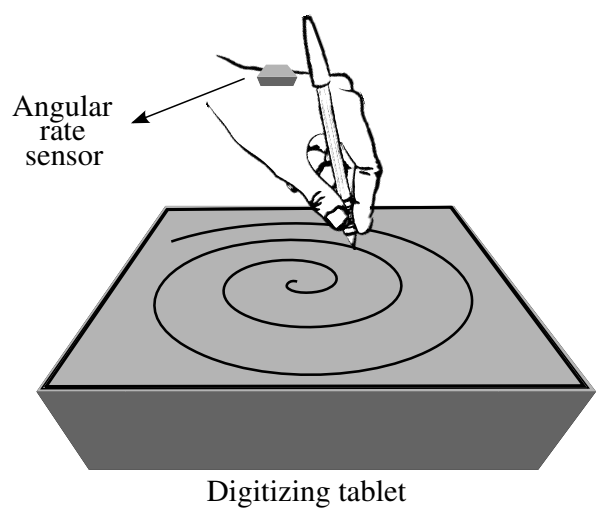

Fig. 1. Graphical representation of the experimental procedure. While the patient performed the task, both the hand motion and the resulted writing were recorder. 


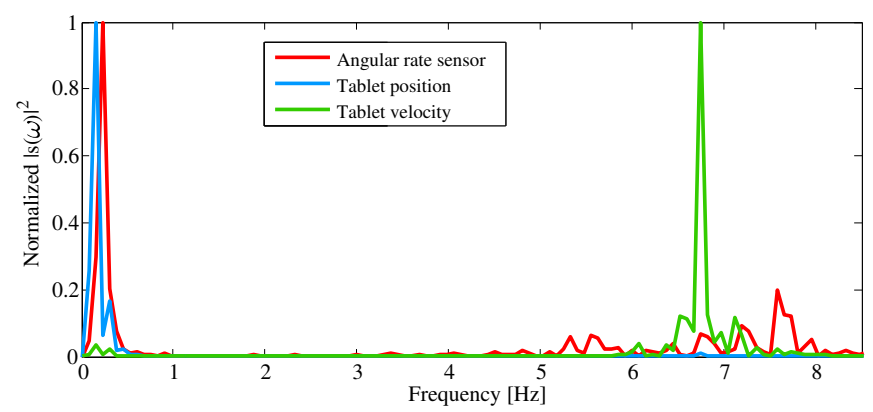

Fig. 2. PSD of both data sets used in this work. For the tablet data, position and velocity in $\mathrm{X}$ are shown.

\section{EXPERIMENTAL EVALUATION}

In this section, we present an experimental evaluation of the proposed algorithm. The task of drawing a spiral was chosen. It is one of the common methods to evaluate tremor severity [1], particularly because loosing his/her writing ability is one of the most serious functional disabilities associated with pathological tremor.

Figure 1 illustrates the experimental setup, where both hand tremor motion and writing data were measured. Commercial sensors were used for both acquisitions. For the inertial measurements, we employed the IDG300, an analog device from Invensense. To record writing data, the Intuos3, a digitizing tablet with 5, 080 lpi resolution from Wacom, was used.

Two different data sets from two patients are analyzed in this paper. In order to evaluate the algorithms performance under different conditions, while in one case the signal from a angular rate sensor was used, in the other data from a digitizing tablet was employed. In figure 2 the power spectrum densities (PSD) of both data sets are shown, which illustrates the assumptions made about the motions frequency ranges. In the analysis conducted within the current section, both data sets were normalized by initial estimates of signal offset. For the presented results, though, the data is de-normalized.

The obtained results are briefly compared with the results provided by the two other cited techniques, the WFLC and the combination of BBF+WFLC. Since a perfect reference voluntary motion is difficult to measure, if not impossible, a quantitative comparison of the methods is difficult to be performed. Hence, the comparison between evaluated methods was made on a visual basis.

Concerning the parameters applied in the algorithms, the same parameters were used for both data sets. The parameters used for the EKF-based algorithm proposed in this paper are shown in the Table I. Voluntary motion has been modeled as a digital low-pass filter with cutoff frequency on $2 \mathrm{~Hz}$. The coefficients are not listed on the table, since they depend on the sampling frequency.

Regarding the method proposed in [6], the same published parameters were used. Parameters used in [2] were not found. Hence, an effort was made to find the parameter $g$ (in [2]) that fitted both data sets, since
TABLE I

PARAmeters USED For OUR EKF-BASEd ALGORITHM.

\begin{tabular}{c|c|c|c|c|c}
\hline$H$ & $\sigma_{s_{t}}^{2}$ & $\sigma_{\omega}^{2}$ & $\sigma_{a_{h}, b_{h}}^{2}$ & $F$ & $\sigma_{s_{i}}^{2}$ \\
1 & $5.10^{-8}$ & $5.10^{-4}$ & $5.10^{-3}$ & 2 & $5.10^{-2}$ \\
\hline
\end{tabular}

the algorithm performance was highly dependent on the data. The value chosen for $g$ was 0.015 and the WFLC parameters were the same taken from [6].

\section{A. Angular rate sensor}

Using a low-cost angular rate sensor attached to a patient's hand, tremor angular velocity was measured while the referred task was performed by the patient. The patient was diagnosed with Parkinson's Disease (PD) and presented variable tremor with unstable amplitude. The sensor was used to measure flexion/extension of the wrist, which was the most severe tremor direction. Tremor frequency was around $7.5 \mathrm{~Hz}$.

Figure 3 shows the acquired data and the estimated voluntary motions. The results provided by the WFLC and the EKF are similar, although the voluntary motion estimated by the WFLC is more noisy, since it is computed directly by $\tilde{s}-\hat{s}_{t}$. The algorithm proposed in [2] was not able to filter effectively the tremor from intentional motion with the gains applied in our work. Smaller $g$ provided better results, but introducing considerable delay if too small gains were applied.

\section{B. Digitizing tablet}

A digitizing tablet is a device commonly used in the clinical evaluation of pathological tremor. It provides two-dimensional measurements of an electronic pen. In this experiment, the patient was told to draw a spiral following a reference drawing. However, we did not assume that the reference spiral could be used as the ground truth for voluntary motion. The data presented here is from a patient diagnosed with essential tremor (ET), who

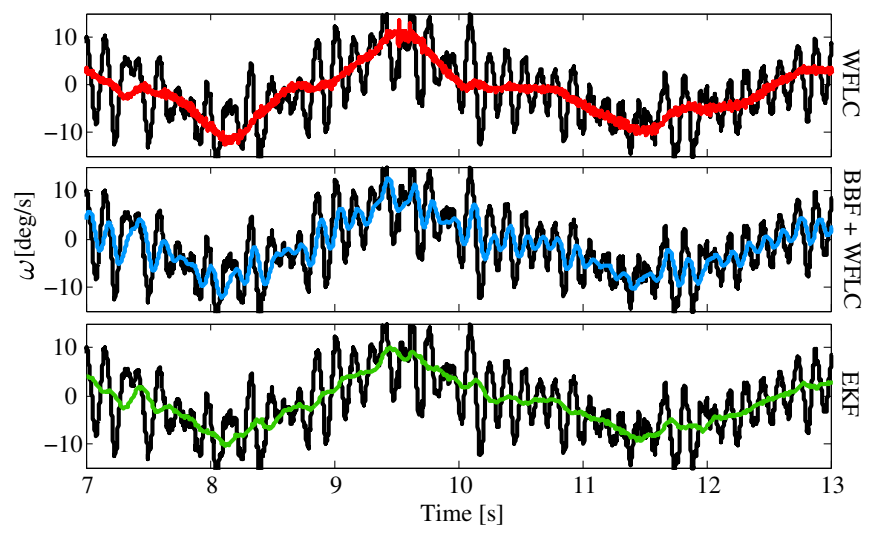

Fig. 3. Angular velocity data (black) and the voluntary motion estimated by our method (green) and the two other evaluated algorithms. 


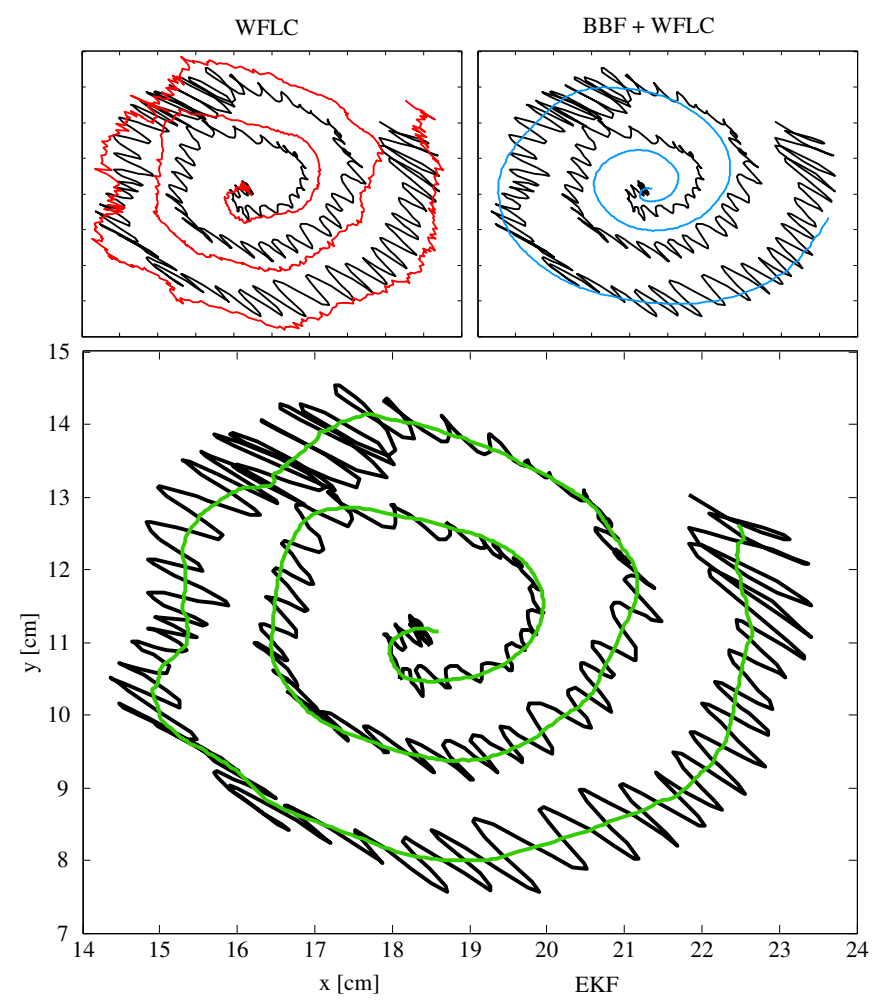

Fig. 4. Spiral drawn by the patient (black) and estimated spiral after filtering trembling motion. The two upper curves results refer to the WFLC and the BBF+WFLC, while the lower graph shows the drawing estimated by the proposed EKF-based method.

had an almost disabling tremor on the dominant hand. Tremor frequency was around $6.8 \mathrm{~Hz}$.

Figure 4 shows both estimated voluntary motions on coordinates $X$ and $Y$. In this case, the differences in performance were greater if compared to results presented on the previous subsection. The WFLC algorithm was not able to correctly estimate tremor, as illustrated by Fig. 5 , and the error was propagated to voluntary motion estimation. Better results were obtained with smaller gains to the FLC phase of the algorithm. With respect to the method BBF+WFLC, the voluntary motion is obtained first from the original signal, $\tilde{s}$. From Fig. 4, it is shown that the result was excessively smoothed and delayed. Better performance was found with higher values for the $g$ parameter. Regarding the results obtained from our EKF-based algorithm, it is illustrated on Fig. 4, that the correct drawing without tremor components is obtained, and on Fig. 5 that voluntary motion was correctly filtered.

\section{Discussion}

In order to analyze the obtained results, it is of great importance to remember that the purpose of this algorithm is to estimate both tremor and voluntary motion from a signal that contains both components. Hence, the algorithm robustness to different motions is an essential feature. In addition, its robustness to signals obtained from different sensors is desirable. In order to compare those features, in both experiments all algorithm parameters in all methods were kept unchanged. The only exception to this rule is the frequency initial estimate.

Within this evaluation framework, while the performance of the EKF-based algorithm was accurate and insensitive to different data sets, this was not the case for the WFLC and BBF+WFLC methods. The same task was executed in the two experiments, but with different sensors sampled with different frequencies. For a particular data set, the parameters of each algorithm could be tuned to provide better results than those presented on Sections III-A and III-B, but it would also decrease the performance in the other experiment, revealing the low robustness of those algorithms. The insensitiveness of the proposed algorithm was greatly due to the assumption of Eq. (4), as the voluntary motion model, and the explicit consideration of process and measurement uncertainties. As for the WFLC and the BBF+WFLC, it was noted that they are not only sensitive to different voluntary motion dynamics and amplitude ratio between tremor and intentional motion, but also to different sampling frequencies. In our comparison, however, care was taken to adjust the algorithm for the right sampling frequency. For the WFLC, for instance, the Butterworth filter applied during the processing was designed accordingly.

The performance of our algorithm with different parameters was not deeply evaluated. The goal was to obtain a sufficiently good performance and compare it with the other methods. Whenever possible, parameters were of the same order from those used in the other methods. $H$, for instance, was set to 1 , since it was the value used in [6]. Also, the algorithms performance in tracking tremor frequency was not compared, since this problem has been mainly addressed in [4]. One possible disadvantage of the presented algorithm is that it is more complex than the other methods. However, considering currently available computational units and the scalar corrections are applied, it is a negligible issue.

Finally, it is important to point out the potential consequences of errors in the estimated tremor and voluntary motions. The applications of the provided estimated motions (outputs of the algorithm presented in this paper)

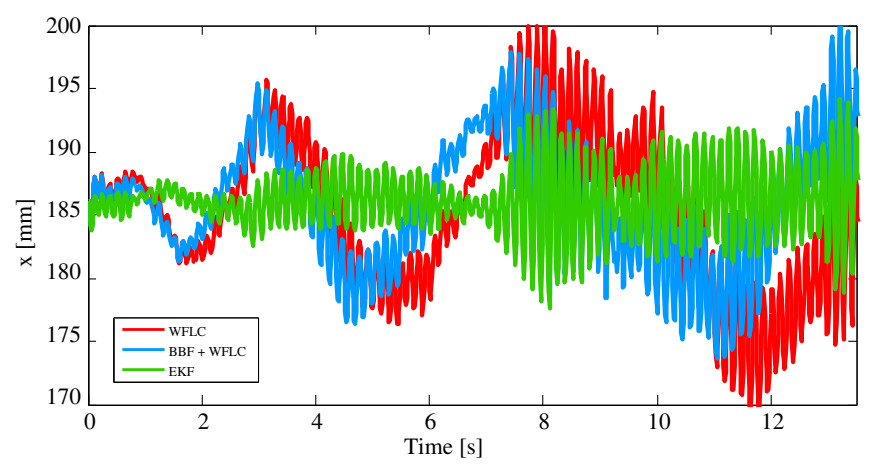

Fig. 5. Tremor in coordinate $\mathrm{X}$ estimated by all three evaluated methods. 
may be used in two classes of problems. Firstly, the estimated intentional motion may be used in applications such as filtering tremor from writing, gesture recognition. In those cases, the overestimation of intentional motion or the existence of high frequency components may prevent the practical use of the measured data.

In a second scenario, the estimated tremor motion may be used as an input to an active tremor compensation system. In this case, a typical application uses an estimate of tremor frequency and amplitude to regulate compensatory action. If tremor is modeled as a first-order harmonic model, tremor amplitude may be given by

$$
P_{t}=\frac{\left\|b_{1}-i a_{1}\right\|^{2}}{4} \text {. }
$$

Within this context, errors in tremor amplitude estimation from tremor data such as those presented in Fig. 5 may also prevent the practical use of such systems.

\section{Conclusions}

For pathological tremor compensation applications, such as a FES-based system, estimation of both tremor and voluntary motions from the resultant composed measured motion is of great importance. In this paper, an algorithm to address this problem is presented. Models are assumed for both motions and the estimation is performed with an EKF.

The performance of the proposed method was evaluated with two experimental data sets from tremor patients performing the same task, drawing a spiral. The motion was measured with two sensors (angular rate and digitizing tablet) and the results were compared with two other methods described in the literature [6], [2]. The proposed method presented a better performance, particularly in terms of robustness to different data sets, being a good candidate for application in real pathological tremor compensation systems.

\section{REFERENCES}

[1] R. J. Elble and W. C. Koller, Tremor. The John Hopkins University Press, 1990

[2] E. Rocon, J. M. Belda-Lois, A. F. Ruiz, M. Manto, J. C. Moreno, and J. L. Pons, "Design and validation of a rehabilitation robotic exoskeleton for tremor assessment and suppression," IEEE Transactions on Neural Systems and Rehabilitation Engineering, vol. 15, no. 3, pp. 367-378, 2007.

[3] A. Prochazka, J. Elek, and M. Javidan, "Attenuation of pathological tremors by functional electrical stimulation I: Method," Annals of Biomedical Engineering, vol. 20, no. 2, pp. 205-224, 1992.

[4] A. P. L. Bó, P. Poignet, F. Widjaja, and W. T. Ang, "Online pathological tremor characterization with Kalman filtering," in 30th Annual International Conference of the IEEE Engineering in Medicine and Biology Society, 2008, pp. 1753 - 1756.

[5] J. G. Gonzalez, E. A. Heredia, T. Rahman, K. E. Barner, and G. R. Arce, "Optimal digital filtering for tremor suppression," IEEE Transactions on Biomedical Engineering, vol. 47, no. 5, pp. 664-673, May 2000.

[6] C. N. Riviere, R. S. Rader, and N. V. Thakor, "Adaptive canceling of physiological tremor for improved precision in microsurgery," IEEE Transactions on Biomedical Engineering, vol. 45, no. 7, pp. 839-846, July 1998.

[7] K. C. Veluvolu, U. X. Tan, W. T. Ang, W. T. Latt, and C. Y. Shee, "Bandlimited multiple fourier linear combiner for real-time tremor compensation," in 29th Annual International Conference of the IEEE Engineering in Medicine and Biology Society, 2007, pp. 2847-50.

[8] D. Simon, Optimal State Estimation: Kalman, H Infinity and Nonlinear Approaches. John Wiley \& Sons, Inc., 2006. 